\title{
Reduced-Port Laparoscopic Surgery for Patients With Proximal Transverse Colon Cancer With Situs Inversus Totalis: A Case Report
}

\author{
Seung-Seop Yeom*, Kyung Hwan Kim, Soo Young Lee, Chang Hyun Kim, Hyeong Rok Kim, Young Jin Kim \\ Department of Surgery, Chonnam National University Hwasun Hospital, Chonnam National University Medical School, Hwasun, Korea
}

Situs inversus is a rare hereditary disorder in which various anomalies have been reported with internal rotation abnormalities. This case involved an 85-year-old woman who had been diagnosed with transverse colon cancer and who underwent reduced-port laparoscopic surgery. All intra-abdominal organs were reversed left to right and right to left. The aberrant midcolic artery was identified during surgery. The total surgery time was 170 minutes, and the patient lost $20 \mathrm{~mL}$ of blood. The patient was discharged on the 8th postoperative day without complications.

Keywords: Situs inversus; Colonic neoplasms; Reduced port laparoscopic surgery

\section{INTRODUCTION}

Situs inversus totalis (SIT) is a rare congenital anomaly with complete inversion of all thoracic and abdominal organs. The incidence rate of SIT is one in 5,000-20,000 people, and it is generally an autosomal recessive genetic condition [1-5]. The surgical approach in these patients is more difficult regarding mirroring the positions of all organs. However, the use of developed instruments, especially in laparoscopic surgery, has led to many successful results in operations on SIT patients [6]. Moreover, reducedport laparoscopic surgery (RPLS), which is single-incision plus

Received: March 21, 2018 - Accepted: May 29, 2018

Correspondence to: Young Jin Kim, M.D.

Department of Surgery, Chonnam National University Hwasun Hospital, Chonnam National University Medical School, 322 Seoyang-ro, Hwasuneup, Hwasun-gun 58128, Korea

Tel: +82-61-379-7642, Fax: +82-61-379-7661

E-mail: kimyjin@chonnam.ac.kr

ORCID code: https://orcid.org/0000-0002-0104-8255

*Seung-Seop Yeom and Kyung Hwan Kim authors contributed equally as co-first authors.

- This article contains Supplemental Video S1.

(C) 2018 The Korean Society of Coloproctology

This is an open-access article distributed under the terms of the Creative Commons Attribution NonCommercial License (http://creativecommons.org/licenses/by-nc/4.0) which permits unrestricted noncommercial use, distribution, and reproduction in any medium, provided the original work is properly cited. one more port laparoscopic approach, may decrease collisions between the operator and the camera holder [7]. We present a case of a patient with SIT and proximal transverse colon cancer who underwent RPLS.

\section{CASE REPORT}

An 84-year-old female visited a local clinic due to a history of hematochezia. Except for high blood pressure and diabetes mellitus, she had no medical or surgical history. Upon colonoscopic examination, a colonic mass was found at the transverse colon. Through colonoscopic biopsy, the colonic tumor was diagnosed as a welldifferentiated adenocarcinoma. The patient was referred for surgical treatment to the Division of Colorectal Surgery at our institution. The physical examination was nonspecific. The laboratory study did not confirm anemia (hemoglobin, $14.9 \mathrm{~g} / \mathrm{dL}$; haematocrit, $43.3 \%$ ), and the serum level of carcinoembryonic antigen was $2.98 \mathrm{ng} / \mathrm{mL}$. A chest X-ray showed dextrocardia and right-sided subphrenic gas in the stomach (Fig. 1). Abdominal computed tomography showed a complete transposition of the intra-abdominal organs, confirming SIT. Furthermore, the colon cancer was situated in the transverse colon and located somewhat proximally (Fig. 2). No metastases were suspected as a result of a positron emission tomography scan of the patient's entire body.

Based on the endoscopic findings above, we performed a laparoscopic hemicolectomy under general anesthesia with the pa- 


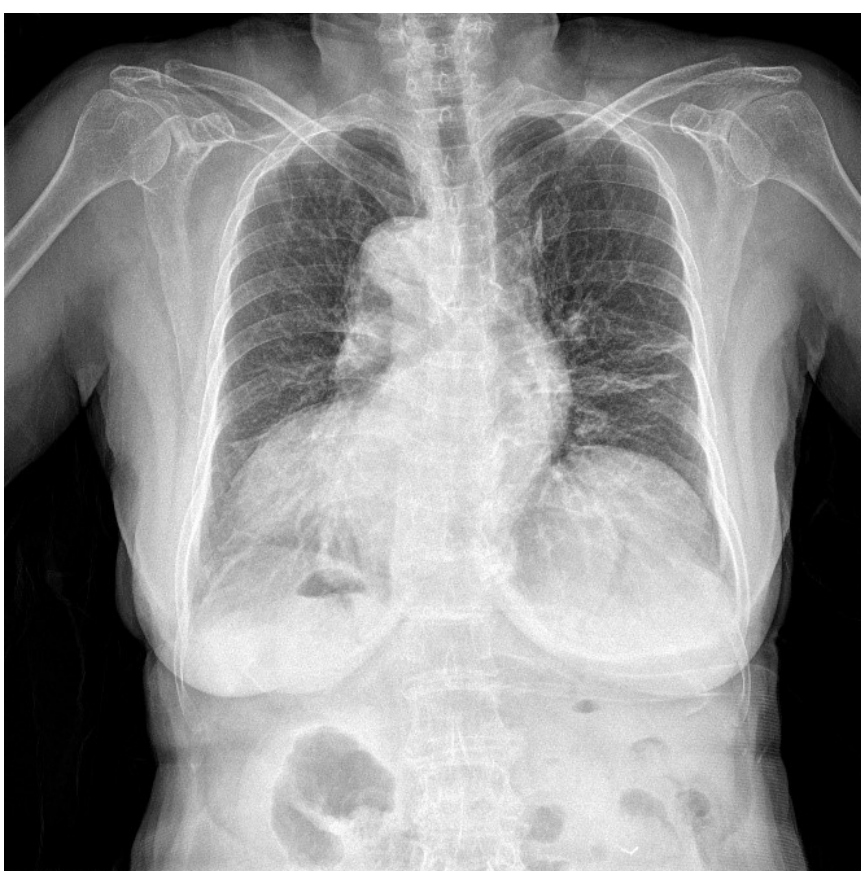

Fig. 1. A chest radiograph showing dextrocardia and a right subphrenic gas in the stomach.

tient in a lithotomy position tilted to the right and with the patient's head down. The surgeon and the camera holder were situated on the right side of the patient, and the first assistant was positioned on the left side. A 4-cm-sized transverse incision, including the umbilicus, was done, and a single-port device (Octoport, Dalim SurgNET, Seoul, Korea) was installed to establish a pneumoperitoneum with an intraperitoneal pressure of up to 12 $\mathrm{mmHg}$. A 5- $\mathrm{mm}$ trocar was placed $10 \mathrm{~cm}$ caudally from the umbilicus (Fig. 3).

Laparoscopic exploration of the abdominal cavity revealed that colon cancer was present at the proximal transverse colon (Supplemental video clip 1); no evidence of peritoneal metastasis was found. All internal organs on the left side and on the right side were reversed as predicted before the operation. After the small intestine and the greater omentum had been placed on opposite sides, a medial to lateral dissection was started. For the D3 lymph node dissection, the ileocecal pedicle was elevated to expose the ileocolic vessels, after which those vessels were ligated with clips at their origins (Fig. 4). Dissection was carried out along the superior mesenteric vein toward the right colic and the mid colic vessels. At the level of the inferior border of the pancreas, 2 arteries originated from the superior mesenteric artery. The smaller one supplied blood to the right-sided colon, and the larger one supplied blood to a more distal area between the distal ascending colon and the mid transverse colon (Fig. 5; right colic and aberrant midcolic artery). The mid colic vein (MCV) and the gastrocolic trunk of Henle (GCT) were found just above those arteries. The

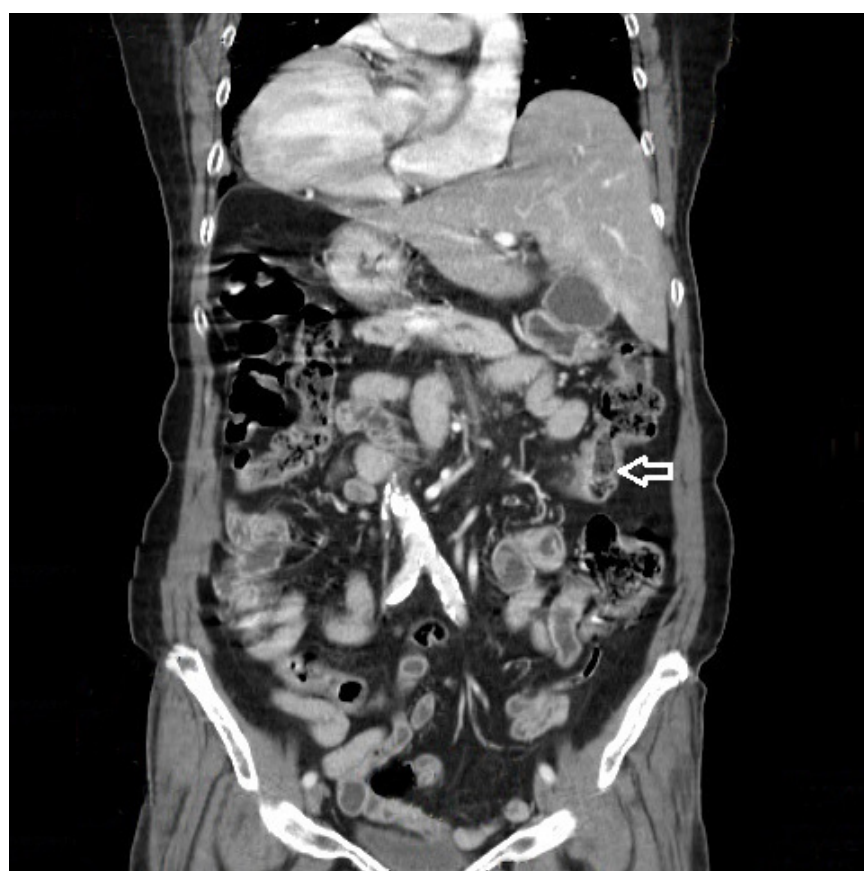

Fig. 2. Computed tomography image showing complete inversion of the abdominal viscera and location of the colon cancer (blank arrow).

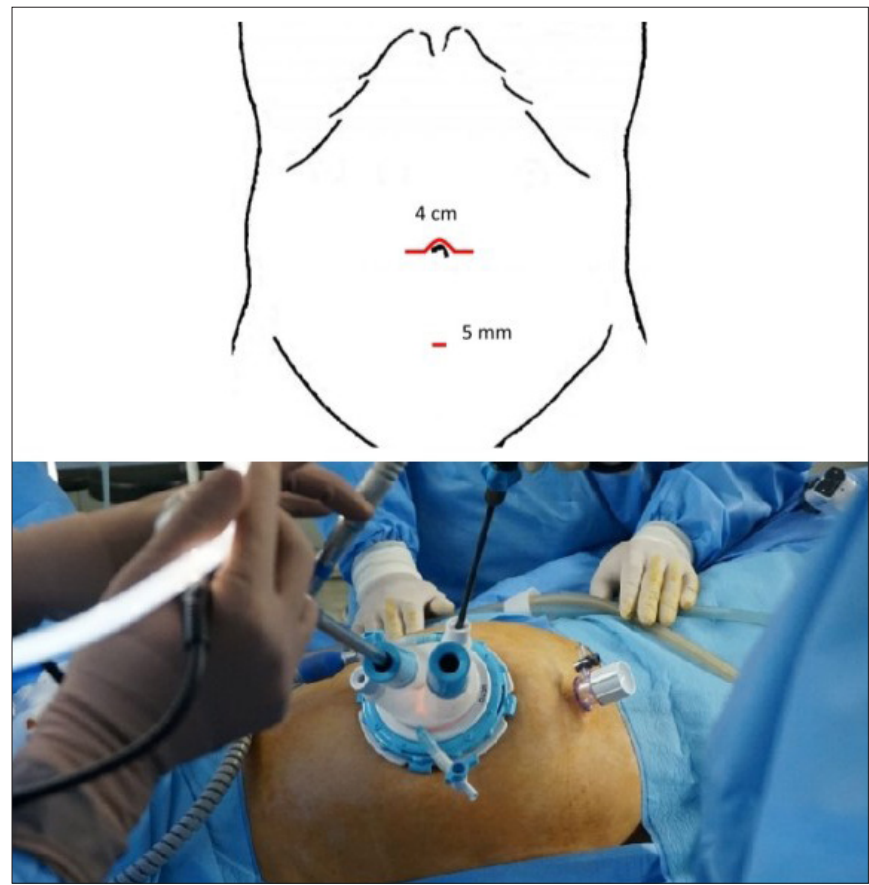

Fig. 3. Trocar placements for reduced-port laparoscopic surgery.

right colic vein (RCV) and the right gastroepiploic vein draining to the GCT were identified, but no other arteries were seen until a lesser sac was opened. The right colic artery, the mid colic artery 


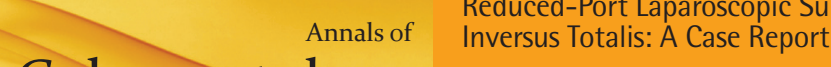 \\ Seung-Seop Yeom, et al.}

Reduced-Port Laparoscopic Surgery for Patients With Proximal Transverse Colon Cancer With Situs

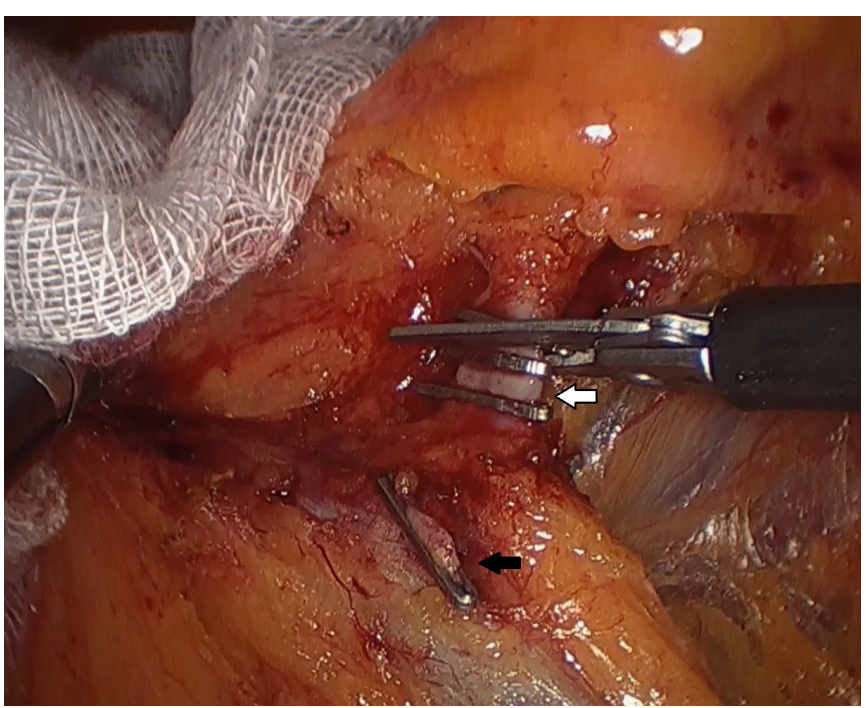

Fig. 4. Division of the ileocolic artery (white arrow) and vein (black arrow).

(MCA), the RCV and the MCV were ligated, and lateral dissection was initiated for colonic detachment from the retroperitoneum. Finally, the greater omentum was divided to the area of the distal margin of colon, including right gastroepiploic arteries, and a remnant retroperitoneal dissection was done.

Through a transumbilical incision, the colon and the ileum were extracted, and the colon cancer was resected with a safety margin. Extracorporeally, a functional end-to-end ileocolic anastomosis was carried out using 2 NTLC 75-mm staplers (Ethicon, Livingston, UK), and the anastomosis was reinforced with seromuscular vicryl 3-0 sutures. The total operative time was 170 minutes, and the total blood loss was $20 \mathrm{~mL}$.

Grossly, the ulcerofungating tumor was located in the transverse colon, and the tumor size was $30 \mathrm{~mm} \times 28 \mathrm{~mm}$. Histologic examination of the resected specimen showed a well-differentiated adenocarcinoma with pericolic soft tissue invasion. No metastasis to 82 isolated lymph nodes had occurred. The final pathologic stage was stage II (pT3N0) according to the seventh edition of the American Joint Committee on Cancer Staging System. The patient's post-operative hospital stay was 8 days, and she was discharged without surgical complications. This case report was approved by the Chonnam University Hwasun Hospital Institutional Review Board (TMP-2018-048) and was eligible for exemption from the requirement to obtain written informed consent from the patient.

\section{DISCUSSION}

SIT is a very rare congenital condition that presents itself in 1 of $5,000-20,000$ people and is inherited in a simple autosomal recessive manner [1-5]. SIT is the complete inversion of thoracic or-

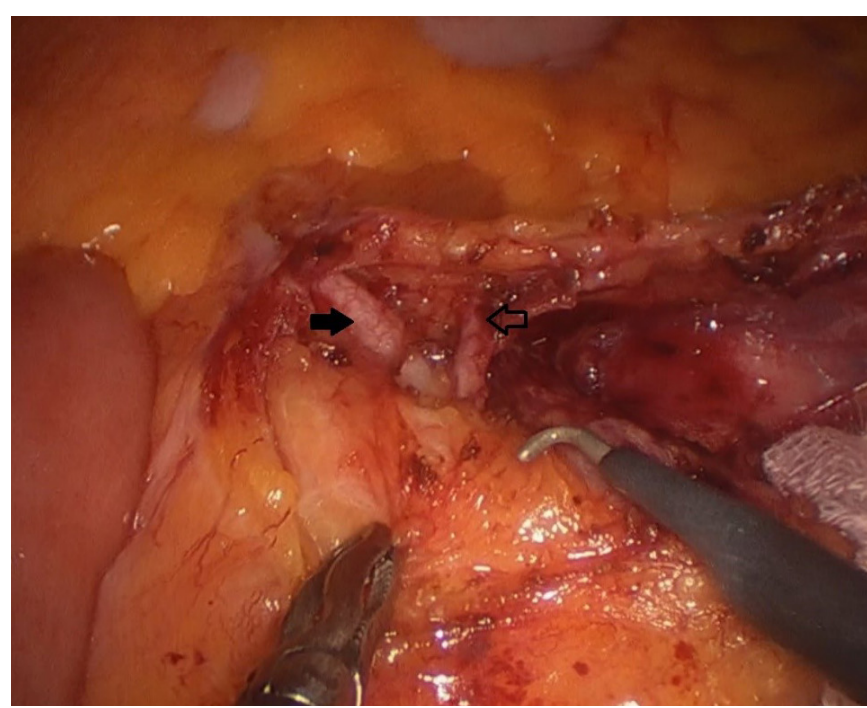

Fig. 5. Aberrant midcolic artery (filled arrow) and right colic artery (blank arrow).

gans and abdominal viscera. Laparoscopic surgery in patients with SIT remains a technical challenge for the surgeon and is relatively rare, with few reported cases. Although surgical techniques are needed to establish a meaningful surgical outcome, several previous reports on laparoscopic surgery for SIT patients confirmed the feasibility of using the laparoscopic approach [1-6].

Single-incision laparoscopic surgery (SILS) for patients with colon cancer is known to be safe and feasible [7-10]. SILS needs a smaller incision than conventional colon cancer surgery, and patients who undergo SILS experience less pain. However, SILS involves difficulties, such as the risk of instrument crowding due to loss of triangulation. RPLS is an alternative method that can be used to avoid this difficulty [9]. Insertion of one more port is not a failure of minimally invasive surgery; rather, it can reduce "sword fighting" between the surgeon and the camera holder [11]. In the present case, an extended right hemicolectomy via RPLS was performed on a patient with SIT. In SIT patients, an inferior vena cava (IVC) interruption and numerous arterial branches, the ipsilateral abdominal aorta and IVC, the intraperitoneal pancreas, polysplenia, and incomplete rotation of intraabdominal organs are reported by radiologists and autopsists $[12,13]$. During the surgery, we also faced difficulties resulting from the mirror-image anatomy and vascular anomalies. We performed the surgery performed via the routine procedure used for patients with normal anatomies, and fortunately, we encountered no significant problems. However, we did experience the "sword fighting" that very often occurs between the camera holder and the holder of a nearby instrument. We found the distant additional 5-mm port to be very useful for avoiding such collisions.

RPLS was a safe and feasible option for this patient with the rare congenital condition SIT. Nevertheless, RPLS should only be per- 
formed on such patients after proper preoperative evaluation to identify combined abnormalities; furthermore, RPLS for such patients should only be performed by an expert laparoscopic surgeon who can rapidly address unexpected situations resulting from the abnormalities.

\section{CONFLICT OF INTEREST}

No potential conflict of interest relevant to this article was reported.

\section{SUPPLEMENTARY MATERIAL}

Supplementary video clip 1 can be found via https://doi.org/10.3393/ac.2018.05.29.1.v001.

Video clip 1. RPLS for a SIT patient.

\section{REFERENCES}

1. Nursal TZ, Baykal A, Iret D, Aran O. Laparoscopic cholecystectomy in a patient with situs inversus totalis. J Laparoendosc Adv Surg Tech A 2001;11:239-41.

2. Fujiwara Y, Fukunaga Y, Higashino M, Tanimura S, Takemura M, Tanaka Y, et al. Laparoscopic hemicolectomy in a patient with situs inversus totalis. World J Gastroenterol 2007;13:5035-7.

3. Jobanputra S, Safar B, Wexner SD. Laparoscopic diverticular resection with situs inversus totalis (SIT): report of a case. Surg Innov 2007;14:284-6.

4. Oms LM, Badia JM. Laparoscopic cholecystectomy in situs inversus totalis: The importance of being left-handed. Surg Endosc
2003;17:1859-61.

5. Huh JW, Kim HR, Cho SH, Kim CY, Kim HJ, Joo JK, et al. Laparoscopic total mesorectal excision in a rectal cancer patient with situs inversus totalis. J Korean Med Sci 2010;25:790-3.

6. Kobus C, Targarona EM, Bendahan GE, Alonso V, Balagué C, Vela S, et al. Laparoscopic surgery in situs inversus: a literature review and a report of laparoscopic sigmoidectomy for diverticulitis in situs inversus. Langenbecks Arch Surg 2004;389:396-9.

7. Bae SU, Baek SJ, Min BS, Baik SH, Kim NK, Hur H. Reducedport laparoscopic surgery for a tumor-specific mesorectal excision in patients with colorectal cancer: initial experience with 20 consecutive cases. Ann Coloproctol 2015;31:16-22.

8. Kawamata F, Homma S, Minagawa N, Kawamura H, Takahashi N, Taketomi A. Comparison of single-incision plus one additional port laparoscopy-assisted anterior resection with conventional laparoscopy-assisted anterior resection for rectal cancer. World J Surg 2014;38:2716-23.

9. Hirano Y, Hattori M, Douden K, Shimizu S, Sato Y, Maeda K, et al. Single-incision plus one port laparoscopic anterior resection for rectal cancer as a reduced port surgery. Scand J Surg 2012;101: 283-6.

10. Choi SI, Lee KY, Park SJ, Lee SH. Single port laparoscopic right hemicolectomy with D3 dissection for advanced colon cancer. World J Gastroenterol 2010;16:275-8.

11. Keller DS, Haas EM. Single-incision laparoscopic colon and rectal surgery. Clin Colon Rectal Surg 2015;28:135-9.

12. Fulcher AS, Turner MA. Abdominal manifestations of situs anomalies in adults. Radiographics 2002;22:1439-56.

13. Kulesza RJ Jr, Kalmey JK, Dudas B, Buck WR. Vascular anomalies in a case of situs inversus. Folia Morphol (Warsz) 2007;66:69-73. 